\title{
First gene therapy for $\beta$-thalassemia approved
}

\author{
Bluebird Bio's gene therapy eliminates the need for blood transfusions in patients with $\beta$-thalassemia.
}

$\mathrm{B}$ luebird Bio has been granted the go-ahead to market its gene therapy for the blood disorder $\beta$-thalassemia. Zynteglo gained conditional market approval from the European Commission in June to treat transfusion-dependent $\beta$-thalassemia in patients 12 years and older who have no other treatment options.

The therapy adds a corrective gene whose product combines with $\alpha$-globin to produce functional hemoglobin, thereby reversing the ineffective red blood cell production seen in $\beta$-thalassemia. This potentially curative treatment sped through the regulatory agency to approval, but the steep price tag- $€ 1.6$ million ( $\$ 1.8$ million) for a treatment course-could prove burdensome for payers and national healthcare providers seeking options for this relatively common recessive disorder. For patients, however, a one-off, potentially curative treatment could be life changing.

The $\beta$-hemoglobinopathies, which include $\beta$-thalassemia and sickle cell disease, are caused by mutations in the $\beta$-globin gene. These gene mutations, of which there are $>200$ known in the population, result in either abnormal hemoglobin structure or reduced or absent $\beta$-globin chains. The clinical manifestations appear several months after birth when gene expression switches from the fetal $\gamma$-globin chain to the adult $\gamma$-chain that forms hemoglobin A ( $\mathrm{HbA})$. The $\beta$-thalassemias vary in severity, but patients with the most severe form, $\beta$-thalassemia major, rely on monthly red blood cell transfusions to survive. Repeated transfusions, however, eventually result in multi-organ damage due to iron overload, which needs daily chelation therapy. Allogeneic hematopoietic stem cell transplantation is a curative option for $\beta$-thalassemia major, when a suitable donor is available. An approach like gene therapy that obviates the need for a matched donor represents a milestone for the field.

Zynteglo is an ex vivo gene therapy that requires the harvesting of a patient's bone marrow stem cells by apheresis (at least $12 \times 10^{6} \mathrm{CD} 34^{+}$cells per kilogram body weight). CD $34^{+}$cells are then transduced ex vivo with the gene encoding $\beta^{\mathrm{A}-\mathrm{T} 87 \mathrm{Q}}$-globin via a BB305 lentiviral vector pseudotyped with vesicular stomatitis virus glycoprotein G. After patients undergo myeloablative preconditioning with busulfan, the transduced CD $34^{+}$cells containing the $\beta^{\mathrm{A}-\mathrm{T} 87 \mathrm{Q}}-$ globin gene are given in a single

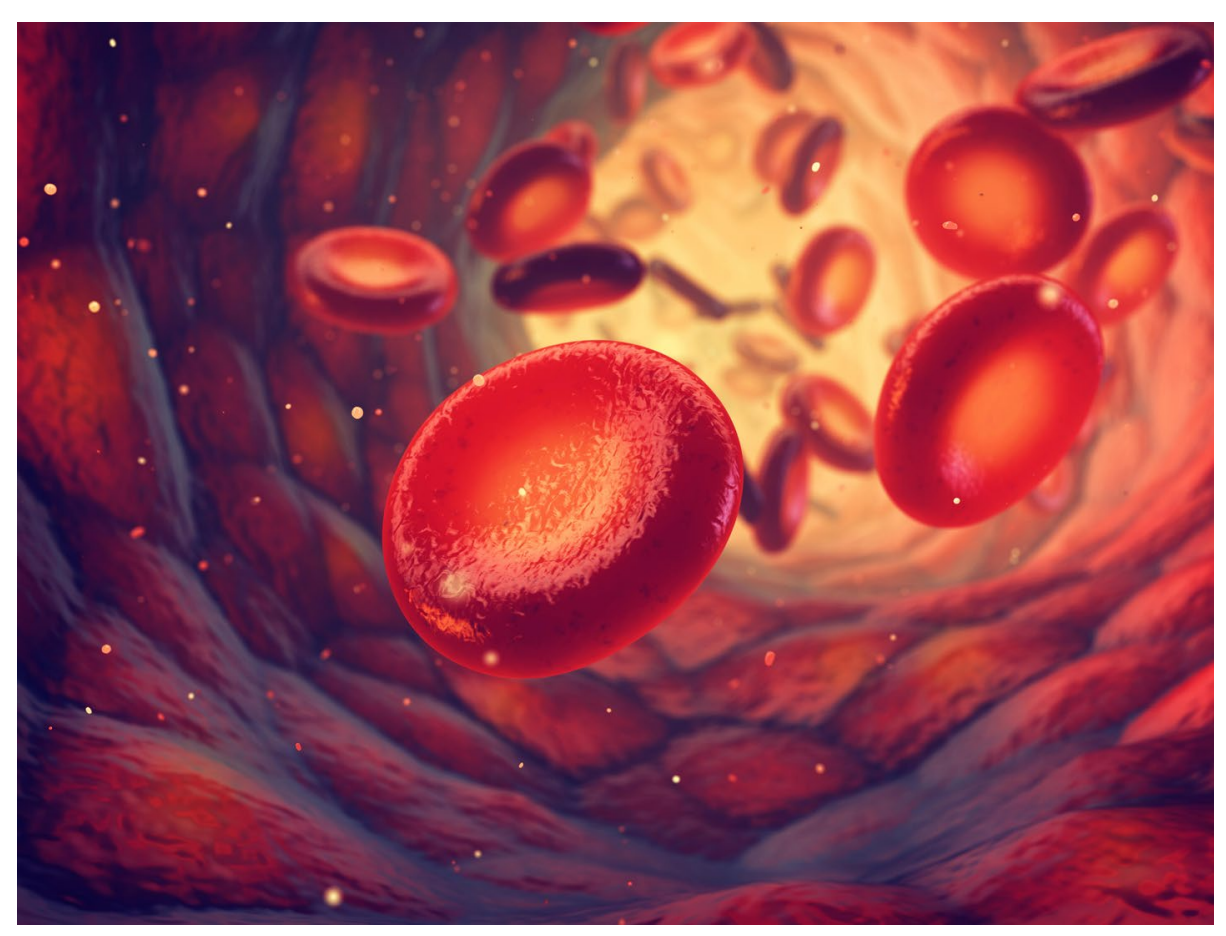

In people with the inherited disorder $\beta$-thalassemia, the oxygen-transport protein hemoglobin in red blood cells is defective. Credit: nobeastsofierce Science / Alamy Stock Photo

injection (minimum dose $5.0 \times 10^{6}$ cells per kilogram), after which they engraft in the bone marrow and differentiate into red blood cells that produce a therapeutic hemoglobin called $\mathrm{HbA}^{\mathrm{T} 87 \mathrm{Q}}$.

The European Commission based its conditional approval on clinical data from 32 adults and adolescents with transfusiondependent $\beta$-thalassemia treated with Zynteglo. In two trials now completed, 11 out of 14 patients reached the primary endpoint of transfusion independence. From two trials that are ongoing, 4 out of 5 evaluable patients no longer needed transfusions. "Becoming transfusionindependent and stopping iron chelation is really a life-changing treatment for patients," says trial physician Marina Cavazzana, a hematologist at the Necker Children's Hospital and INSERM, Paris, France. "For example, after the therapy, patients were able to take part in physical activity that would have been impossible before therapy."

It's still early days, though. The approval trials followed patients for only 2 years, and individuals were considered transfusion independent if they did not need transfusions for 12 months or more and were still independent by the end of the study. "Although these are very successful results, we need to better understand the long-term sustainability of the response," says Maria Domenica Cappellini, a clinician at the University of Milan and the Maggiore Policlinico Hospital, Milan, Italy. Most clinicians, including Cappellini, expect that a patient who has been transfusion-free for 12 months will remain this way for many years; to confirm this, Bluebird is following patients from the trial for another 13 years.

Zynteglo is not approved for the most severe type of $\beta$-thalassemia. This is because, according to data that were not included in the approval package, five out of eight patients with the severest form (the $\beta^{0} / \beta^{0}$ genotype) treated with Zynteglo had to return to blood transfusions. When this information became public, Bluebird's share price crashed. Since then, the company has refined its approach. "The trials really helped the field understand some of the limitations of gene addition therapy," says Stefano Rivella, a pediatrician at the Children's Hospital of Philadelphia and University of Pennsylvania. For the benefit to manifest in $\beta^{0} / \beta^{0}$ patients with the most 
severe disease, a higher percentage of blood cells carrying the transgene sequences was required. Bluebird also had to improve the expression levels and ensure more than one vector integrated per genome.

Bluebird has used this improved vector design and transduction process for the two ongoing trials, which include patients with the most severe genotype. To date, the one evaluable patient achieved transfusion independence. Bluebird intends to use data from these trials to provide the more comprehensive benefit-risk data that are needed to switch the European Union conditional approval to standard marketing authorization, and for a US Food and Drug Administration (FDA) filing anticipated in 2020.

Another $\beta$-globin gene therapy in clinical trials is Orchard Therapeutics' OTL-300, which uses the GLOBE lentiviral vector encoding wild-type $\beta$-globin that is administered by intrabone injection. In its trial of OTL-300, transfusion requirements were reduced in the three adult participants, and three out of four pediatric participants stopped transfusions. Investigators noted that a younger age and a higher copy number were linked with a better outcome. Despite the improvements, clinicians expect more. "Gene therapy must be a cure," says Cappellini. "Transfusion reduction could be achieved with other therapeutic approaches for $\beta$-thalassemia that are in ongoing trials."

The prospect of disease correction is alluring, but gene therapy carries its own risks. Before administration of gene therapy, patients undergo grueling chemotherapy with busulfan to wipe out their own mutant cells and ensure that a high proportion of the gene-modified CD $34^{+}$cells engraft in their bone marrow. Myeloablation can cause infertility, and the longer term effects are unknown. New vectors can help reduce some of the risks and side effects and improve therapeutic safety. "A stronger vector that expresses higher levels of hemoglobin per integration site might be able cure the disorder without the need for complete myeloablation," says Rivella.

Manufacturing this type of product for roll-out in the clinic remains a major challenge, something that became apparent the trials leading up to the approval. Once patient cells were harvested, they were sent to a central manufacturing facility for enrichment and modification with Zynteglo before being sent back to the clinical trial site."The requirement for centralizing the process is a huge obstacle to the wider use of this gene therapy," says Cavazzana. To try to circumvent this issue, she is working with industrial partners to develop an on-site automated closed system that does not require good manufacturing practice (GMP)-compliant facilities. "The need for GMP production is really the bottleneck in the whole process," she adds.

Zynteglo is also in trials for sickle cell disease. Like $\beta$-thalassemia, sickle cell disease is a monogenic disorder, characterized by a single mutation in the $\beta$-globin gene, the $\beta \mathrm{S}$ mutation, that results in a single amino acid substitution at codon 6 . The amino acid substitution prompts the modified $\beta$-globin chains to form polymers, which make the red blood cells rigid and prone to occluding vasculature. Gene-addition therapies in sickle cell disease aim to dilute out the defective protein that distorts the red blood cells into a sickled shape. Hematopoietic stem cell transplantation from a matched donor is the only curative therapy, but again is not an option for the majority of patients.

Early results from Zynteglo in sickle cell trials are promising. In the four patients with at least 12 months of follow-up, $\beta^{\mathrm{A}-\mathrm{T} 87 \mathrm{Q}_{2}}$ derived hemoglobin production increased to $\geq 50 \%$ of total hemoglobin, and symptoms such as acute chest syndrome and serious vaso-occlusive crisis were eliminated. Another way of diluting out defective hemoglobin is to use fetal $\gamma$-hemoglobin-which has antisickling properties. Aruvant Sciences' gene therapy RVT-1801, which uses an ex vivo lentiviral vector to deliver a modified fetal $\gamma$-hemoglobin gene to a patient's purified CD $34^{+}$cells, reduced vaso-occlusive crises in two treated patients with sickle cell disease.

Another genome editing approach is to reactivate fetal hemoglobin. This can be achieved by suppressing the expression of the BCL11a gene, which encodes a protein involved in the switch from $\gamma$ - to $\beta$-globin in erythroid progenitor cells. CRISPR Therapeutics and Vertex use CRISPR-Cas9 technology to repress $B C L 11 a$ in their investigational therapy CTX-001 for sickle cell disease and $\beta$-thalassemia, whereas Sangamo uses gene editing with zinc-finger nuclease technology to make the genetic changes in ST400, a therapy in development for $\beta$-thalassemia. The first patient treated with ST 400 , who had a $\beta^{0} / \beta^{0}$ genotype, did not need transfusions during 5 weeks of follow-up. Bluebird is using another method to silence BCL11a: a lentiviral vector containing short hairpin RNA (shRNA) as an RNA interference gene therapy. With this method, Bluebird reduced BCL11A expression by about $90 \%$ and eliminated irreversibly sickled cells in one patient, according to data presented at the American Society of Hematology in 2018.

Pharmacological approaches to raise hemoglobin levels are also forging ahead. Fusion proteins known as ACEs, which contain the extracellular domain of the activin receptor linked to the Fc portion of human IgG1, are under also investigation. These ligand traps act on the transforming growth factor- $\beta$ (TGF $\beta$ ) superfamily to increase late-stage erythropoiesis. Acceleron Pharma's fusion sotatercept improved hemoglobin levels and reduced transfusion requirements in an open-label dosefinding study in $\beta$-thalassemia. And in June, the FDA accepted a biologics license application from Celgene and Acceleron for luspatercept, a ligand-trap drug to treat $\beta$-thalassemia and myelodysplastic syndrome-associated anemias.

"The fact that there are both gene therapy and pharmacological drugs is really important," says Rivella. Drugs such as luspatercept could be enough to stop transfusions in patients who have a less severe type of $\beta$-thalassemia, known as thalassemia intermedia, who still have some expression of $\beta$-globin.

Drugs in the pipeline for sickle cell disease include Global Blood Therapeutics' voxelotor, an oral small-molecule therapy that inhibits hemoglobin polymerization, which is in phase 3 trials. Novartis submitted a biologics license application to the FDA for crizanlizumab, a humanized IgG2 monoclonal antibody against CD62 (also known as P-selectin) that reduces cell-cell interactions, which is hoped to reduce vasoocclusion. Rivipansel from GlycoMimetics and Pfizer, a small-molecule pan-selectin inhibitor based on sialyl Lewis X, however, failed phase 3 trials in August.

Pharmacological therapies may benefit patients in lower-income countries or lowerincome populations. Even in higher-income countries, gene therapies are a financial challenge. Bluebird intends to use a payfor-performance plan (Nat. Biotechnol. 37, $697,2019)$, with initial payment of $€ 315,000$ $(\$ 348,000)$ and four additional annual payments, each of that amount, provided the therapy continues to be effective-for a total of $€ 1.58$ million ( $\$ 1.8$ million) per patient. Karl Claxton, Professor of Economics at the University of York and former member of the United Kingdom's National Institute for Health and Care Excellence (NICE) Appraisal Committee, believes this is pricing is overly optimistic. "The company are going to offer some pretty substantial discounts and rebates over and above their pay-for-performance model for the therapy to be deemed cost-effective by governmentprovided healthcare systems," he says.

\section{Charlotte Harrison \\ Canterbury, UK}

Published online: 9 September 2019

https://doi.org/10.1038/d41587-019-00026-3 